\section{Low-dimensional models of subcritical transition to turbulence}

\author{
Jeffrey S. Baggett and Lloyd N. Trefethen \\ Center for Applied Mathematics, Cornell University
}

Abstract. In the past five years, working largely independently, five groups of researchers have proposed lowdimensional models of the behavior of parallel shear flows at high Reynolds numbers. These models are compared, and it is found that they are more similar than their authors have recognized. Among other similarities, most of them exhibit a threshold amplitude $\epsilon=O\left(R^{\alpha}\right)$ as $R \rightarrow \infty$ for some $\alpha<-1$, where $R$ is the Reynolds number, for perturbations of the laminar state that may excite transition to turbulence.

\section{INTRODUCTION}

Certain laminar fluid flows undergo transition to turbulence in a manner that cannot be explained by traditional linear stability theory. Incompressible flow in a circular pipe is the simplest example, and plane Couette flow, between two parallel plates moving relative to one another, is another. Such flows are stable to infinitesimal perturbations, regardless of the Reynolds number $R$, yet if $R$ is high enough, they invariably become turbulent. Resolving this paradox is a longstanding problem of fluid mechanics [7].

The resolution that we and many others favor is as follows. For any fixed $R$, it is true that no infinitesimal perturbation of the laminar flow can excite transition to turbulence. If $R$ is large, however, a finite perturbation of exceedingly low amplitude may be enough to excite transition. Specifically, let us define $\epsilon$ to be the minimum amplitude of all disturbances that may excite transition (measured in the energy norm). In the past five years, evidence based on simple models and on Navier-Stokes simulations has been mounting that for the flows of interest, $\epsilon$ may shrink rapidly as $R \rightarrow \infty$. For a given flow geometry, it may be expected that $\epsilon$ and $R$ will be related by a law approximately of the form

$$
\epsilon=O\left(R^{\alpha}\right)
$$

as $R \rightarrow \infty$. In [23] it was conjectured that for plane Couette and related flows, (1) holds for some $\alpha$ strictly less than -1 . Numerical simulations have subsequently borne out this conjecture, suggesting threshold exponents at least as low as $-5 / 4$ for plane Couette flow and $-7 / 4$ for plane Poiseuille flow $[12,17]$. (In the latter case the number refers to transition by routes unrelated to the linear Tollmien-Schlichting instability.)
The organizing principle of this paper is the question,

$$
\begin{gathered}
\text { What is the threshold exponent } \alpha \\
\text { for transition to turbulence? }
\end{gathered}
$$

In this phrasing of the problem, we intend for $\alpha$ to represent the minimal exponent such that (1) is satisfied. (If the relationship of $\epsilon$ and $R$ is more complicated than just a power law, involving logarithms or other complications, $\alpha$ can be defined as the infimum of all exponents for which (1) holds.) Of course, the threshold exponents may differ for different flow geometries. Our explanation of the phenomenon of subcritical transition is that for the geometries where this phenomenon occurs, $\alpha$ is substantially less than 0 , making laminar flows at high Reynolds numbers in practice unstable, since even the most careful laboratory experiment must introduce some small finite perturbations to the ideal flow.

The idea that the limits to stability may diminish as $R \rightarrow \infty$ is an old one, going back at least to Lord Kelvin in 1887 [14]. On the other hand, the formulation of this idea in terms of threshold exponents, so far as we know, first appeared in [23]. In that paper, the conjecture $\alpha<-1$ was motivated by a simple model consisting of a system of two ordinary differential equations representing non-modal linear amplification coupled with nonlinear mixing.

The purpose of this paper is to present some rather surprising results concerning low-dimensional models of parallel shear flows. In the past five years a number of authors have proposed such models. One set comes from our own group at Cornell University:

$$
\begin{aligned}
& \text { TTRD }= \text { Trefethen, Trefethen, Reddy and Driscoll, } \\
& 1993[23] \\
& \text { BDT }=\text { Baggett }, \text { Driscoll and Trefethen, } 1995[1] \\
& \text { BT }=\text { Baggett and Trefethen, 1995, unpublished }
\end{aligned}
$$

Another is due to Waleffe at MIT, an outgrowth of earlier work with Hamilton and Kim at the Center for Turbulence Research at NASA Ames Research Center and Stanford University [10,26]:

$$
\mathrm{W}=\text { Waleffe, } 1995[24,25]
$$

A third comes from the University of Marburg in Germany, building upon earlier work in Marburg by Boberg and Brosa [2]:

$$
\mathrm{GG}=\text { Gebhardt and Grossmann, } 1994 \text { [8] }
$$

A fourth has been described in a paper from the Royal Institute of Technology in Stockholm,

$$
\begin{aligned}
\mathrm{KLH}= & \mathrm{G} . \text { Kreiss, Lundbladh and Henningson, } \\
& 1994[15]
\end{aligned}
$$

Finally, a fifth model has been proposed in a manuscript from Göteborg, Sweden,

$$
\text { JRB = Johnson, Rannacher and Boman, } 1995 \text { [13] }
$$

One might think that these five groups of models would have been compared to one another, but for the most part this is not so. Part of the reason for this is that 
they were devised for disparate purposes. The models TTRD, BDT, BT, and GG were developed to shed light on the process of transition from low-amplitude perturbations, starting from the view that non-normal linear effects are the essence of the phenomenon and that nonlinear details are less critical. The model $\mathrm{W}$ was developed to shed light on the structure of turbulent boundary layers, and the opposite view was taken that the essential point is to get the nonlinearities right. Indeed, in [24] and [25] it is asserted heatedly that the $\mathrm{W}$ and TTRD/BDT approaches are contradictory. The model $\mathrm{KLH}$ was developed in part for technical mathematical reasons. The model JRB was motivated by the problem of error control in computational fluid dynamics.

We have compared the models listed above. In particular, though most of them were designed for purposes unrelated to $(*)$, we have investigated the threshold exponents $\alpha$ that they exhibit. We find that the mathematical features of these models are all unexpectedly similar. All involve linear, non-normal amplification coupled with nonlinear mixing. Moreover, the threshold exponents they produce are similar, being $\alpha=-3$ for most of those models that we describe as being without a key "selection rule" and $\alpha=-2$ for most that do have this selection rule. This agreement of exponents is particularly striking in view of the fact that two of the authors of these models informed us on occasions in the past that the idea $\alpha<-1$ was a foolish one, not consistent with a correct understanding of the physics!

What do these low-dimensional models tell us about actual fluid flows? In the final two sections we consider this matter briefly.

\section{FUNDAMENTALS}

Subcritical transition to turbulence is a phenomenon most commonly associated with parallel shear flows. In particular, two flows exhibit this phenomenon in its purest form (Fig. 1). One is pipe flow, also known as HagenPoiseuille flow, in an infinite circular pipe. In this case, regardless of the Reynolds number $R$, the laminar state (parabolic velocity profile) is linearly stable, yet transition to turbulence is typically observed for $R \approx 2000$ or larger. In an exceptionally careful experimental situation, laminar flows can be maintained with $R>10^{4}$, and the record is on the order of $10^{5}$, but this is highly unusual. The other is plane Couette flow, in an infinite channel bounded by two parallel plates moving at constant speed relative to one another. The situation here is much the same. The laminar state (linear velocity profile) is stable for all $R$, but transition is typically observed for $R \approx 350$ or larger [6,18,22]. The "record" experimental values of $R$ for laminar flows are not so high in this case, presumably since experiments are more difficult and rarer.

Fig. 2 is a heuristic bifurcation diagram that summarizes the problem. For any $R$, the laminar state is a stable fixed point of the incompressible Navier-Stokes

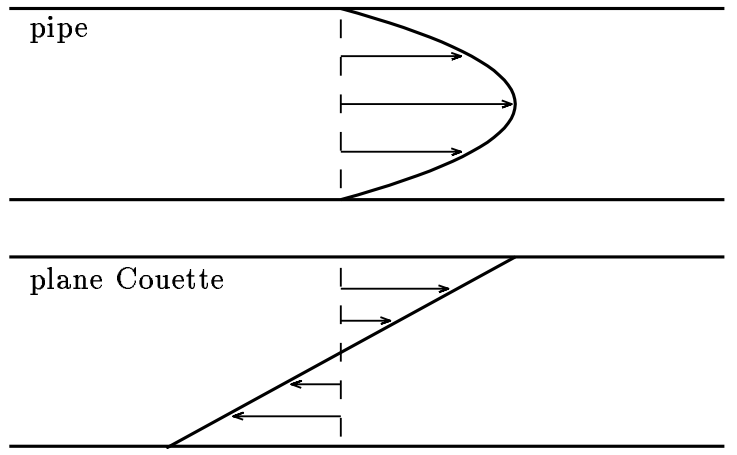

FIG. 1. Laminar velocity profiles for pipe and plane Couette flows. These solutions of the Navier-Stokes equations are stable to infinitesimal perturbations in principle for all Reynolds numbers $R$, but unstable in practice for large $R$.

equations. This state is represented by the dark line along the $R$ axis, corresponding to zero perturbation from the laminar state. Somewhere in phase space, however, there is also a turbulent state to which flows tend to be attracted. Sufficiently small perturbations necessarily relaminarize, but larger perturbations may jump to the turbulent state. The boundary between these behaviors is suggested by the dashed line.

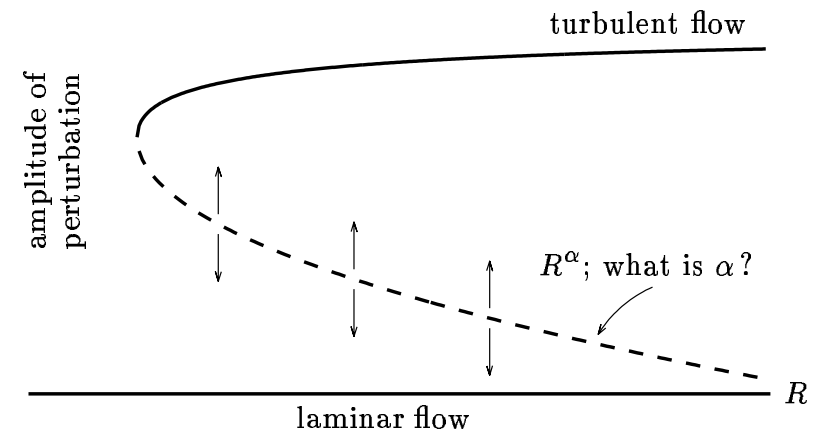

FIG. 2. Heuristic bifurcation diagram for subcritical transition to turbulence. The basin of attraction of the laminar state shrinks rapidly as $R \rightarrow \infty$. Small deviations from the laminar flow are enough to move the flow across the boundary into the basin of attraction of the turbulent state.

We emphasize that Fig. 2 is only heuristic. The true state space is infinite dimensional, so one must not think of the dashed line, for example, as representing a smooth manifold that approaches the axis in a simple fashion. On the contrary, it represents the minimum distance from the origin to some infinite-dimensional manifold of presumably great complexity. Perturbations of the laminar flow of amplitudes far larger than $R^{\alpha}$ may also lie in the basin of attraction of the laminar state, if they happen to lie in directions that are not effective at exciting transition.

The existence of a mathematically well-defined "turbulent state," as assumed in our remarks above and in Fig. 2, may be questioned. Brosa [3] and Crutchfield and Kaneko [5] have argued that the turbulence that is ob- 
served in such flows may in principle consist not of an invariant set such as a strange attractor, but of a combination of transient phenomena (perhaps exponentially or doubly exponentially long ones) excited by perturbations. This possibility is intriguing, and it introduces a potential complication into the task of defining $(*)$ in a rigorous way. But we shall not pursue it here, as it is of little significance to what is observed in experiments or numerical simulations. In practice, at sufficiently high Reynolds number, turbulence is unavoidable, and permanent once it arrives.

Pipe and plane Couette flows are not the only flows of concern in this paper. Other shear flows too exhibit subcritical transition to turbulence, of which the best known examples are plane Poiseuille flow (between two stationary infinite parallel plates) and Blasius boundary layer flow (along a flat wall). In both cases, the picture of Fig. 2 must in principle be modified. Consider plane Poiseuille flow, for example. Here, there is a linear instability for $R>5772$, a Tollmien-Schlichting wave, which would appear in Fig. 2 as a bifurcation point on the $R$ axis [7]. Nevertheless, in practice the transition to turbulence of plane Poiseuille flows appears to follow much the same course as that of pipe and plane Couette flows. It is finite-amplitude 3D disturbances that excite transition in most experiments, not Tollmien-Schlichting waves, and this happens both above the critical value 5772 and well below it. Thus the question $(*)$ is of interest also for plane Poiseuille flow, though a rigorous formulation of it would necessitate qualifications such as a condition of transition on a short (convective) rather than long (diffusive) time scale.

\section{THE FIRST CORNELL MODEL}

We begin by discussing the models developed by our own group at Cornell. These arose from studies of hydrodynamic stability. In the late 1980s and early 1990s, it was discovered by various researchers that for certain linearly stable flows, transient amplifications of flow perturbations by factors of hundreds may be introduced by mechanisms that are linear but non-modal, that is, unrelated to eigenvalues $[2,4,9,19,23]$. This raised the question of how such mechanisms might bring about transition to turbulence, when combined with the nonlinear interactions of the Navier-Stokes equations.

In [23] we proposed the view that the transition process is "essentially linear" in the sense that its qualitative features are not sensitive to the details of these nonlinear interactions. Our proposal was that the role of nonlinearity in transition is to serve as a mixing mechanism, enabling outputs from the linear, non-modal amplification process to be recycled back to inputs, as suggested in Fig. 3a. According to this view, even if the NavierStokes equations happened to have quite different nonlinear terms than the actual ones, there would probably still be a recognizable phenomenon of subcritical transition to turbulence. (The same view has been propounded (a) Marburg/Cornell

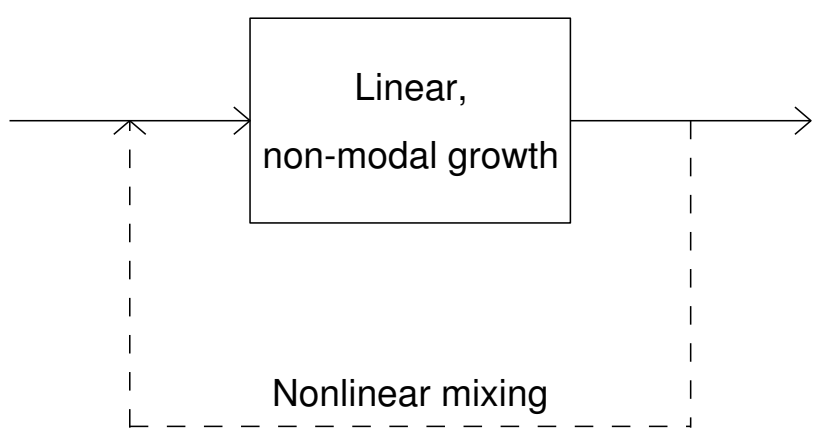

(b) MIT/Sweden

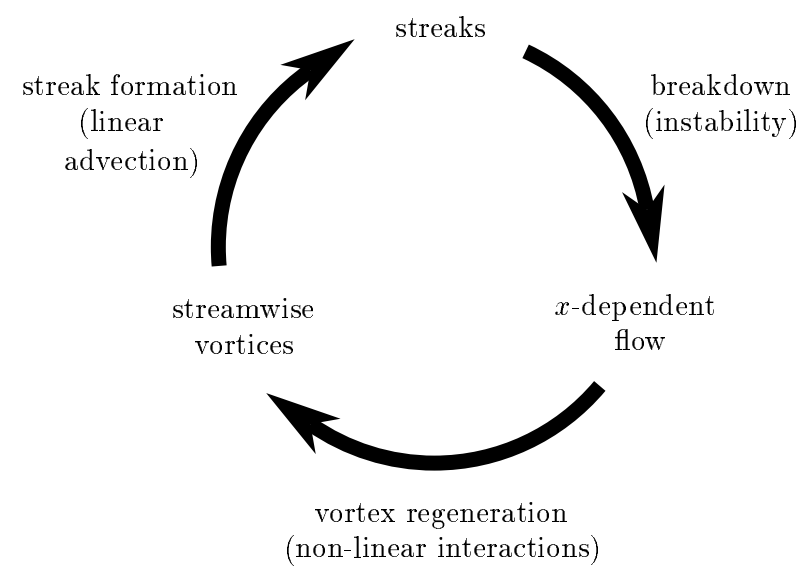

FIG. 3. Schematic illustration of the ideas underlying (a) the Marburg and Cornell models (figure taken from [1]) and (b) the MIT and Swedish models (figure taken from [10]). Schema (a) is abstract, depending only on certain general mathematical properties, whereas schema (b) is physical, attempting to delineate the actual structures that interact in shear flows at high Reynolds number. Apart from this difference, however, these ideas correspond more closely than it may at first appear. The upper-left arrow in the MIT/Sweden schema represents the dominant mechanism of what the Marburg/Cornell schema calls "linear, non-modal growth," and the other two arrows amount to a proposal of a dominant mechanism of "nonlinear mixing". It might appear that the division of this latter part of the loop into two arrowsbreakdown followed by regeneration-represents a fundamental difference between (a) and (b), but this is not so. When "nonlinear mixing" is modeled by arbitrary quadratic interactions among variables, these two phases usually still emerge, for reasons explained in Sec. 9.

by the Marburg group; see Sec. 6.)

To elucidate this idea, we proposed a two-variable model:

$\left(\begin{array}{l}u \\ v\end{array}\right)^{\prime}=\left(\begin{array}{cc}-R^{-1} & 1 \\ 0 & -2 R^{-1}\end{array}\right)\left(\begin{array}{l}u \\ v\end{array}\right)+\left\|\left(\begin{array}{l}u \\ v\end{array}\right)\right\|\left(\begin{array}{cc}0 & -1 \\ 1 & 0\end{array}\right)\left(\begin{array}{l}u \\ v\end{array}\right)$,

where ' denotes differentiation with respect to $t$ and $\|\cdot\|$ is the 2-norm (square root of sum of squares). The presence of distinct coefficients $-R^{-1}$ and $-2 R^{-1}$ along the 


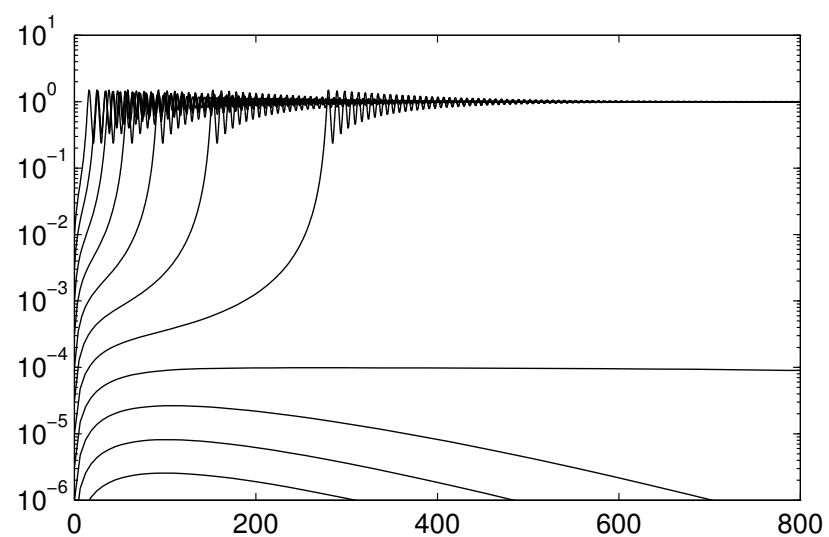

FIG. 4. Evolution curves for the model (TTRD) (threshold exponent $\alpha=-3)$ with $R=100$. The curves show the vector norm $\|y(t)\|$ for initial conditions of amplitudes $\|y(0)\|=$ $10^{-7}, 10^{-6.5}, 10^{-6}, \ldots, 10^{-2}$.

diagonal of the first matrix, however, is of little significance to the behavior of this system (the coefficients were made distinct so that readers would not be distracted by nondiagonalizability), so let us immediately simplify the equations to the following form:

$$
\left(\begin{array}{l}
u \\
v
\end{array}\right)^{\prime}=\left(\begin{array}{cc}
-R^{-1} & 1 \\
-R^{-1}
\end{array}\right)\left(\begin{array}{l}
u \\
v
\end{array}\right)+\left\|\left(\begin{array}{l}
u \\
v
\end{array}\right)\right\|\left(\begin{array}{l}
-1 \\
1
\end{array}\right)\left(\begin{array}{l}
u \\
v
\end{array}\right) .
$$

Here and throughout this paper, blank matrix entries are zero. In this system of differential equations, the first term represents a linear, non-modal transient amplifying process of amplitude $O(R)$ and time scale $O(R)$. The second term mixes the two variables nonlinearly but does not directly affect the energy, since the matrix involved is skew-symmetric.

Fig. 4, based on Fig. 10 of [23], gives an indication of the behavior of (TTRD) for $R=100$. In this paper we shall present a number of figures of this kind, always following the same format. Eleven curves are shown, corresponding to $\|y(t)\|$ as a function of $t$ for eleven different initial conditions $y(0)$, where $y$ represents the vector of dependent variables (here, $y=(u, v)^{T}$ ). The initial conditions have norms $\|y(0)\|=10^{-7}, 10^{-6.5}, 10^{-6}, \ldots$, $10^{-2}$. In Fig. 4, as in our other analogous figures, one sees that the lower curves have approximately the same shape, differing only in vertical displacement. This is because in these cases, the amplitudes are too low for the nonlinear terms to have much effect. What remains is just the linear, non-modal behavior: amplification by about 1.5 orders of magnitude followed by slow decay to the zero state analogous to laminar flow. The upper curves, however, are strongly affected by nonlinearity. At the beginning, they follow the shape of the linear evolution, but in the cases with $\|y(0)\|>10^{-5.5}$, they are attracted as $t \rightarrow \infty$ to a state of magnitude $O(1)$, analogous to turbulent flow.

What is important in Fig. 4 is that although the linear amplification is by less than two orders of magnitude and the nonlinear terms conserve energy, the ultimate amplification in the nonlinear system is by close to six orders of magnitude. This is the phenomenon called "bootstrapping." Specifically, for this particular model with $R=10^{2}$, the threshold amplitude for transition is about $3 \times 10^{-6}$, and this figure decreases with exponent $\alpha=-3$ as $R \rightarrow \infty$. We shall not present the evidence for this and other statements about exponents in this paper, but we assure the reader that in every case, numerical experiments show that the $R^{\alpha}$ dependence is clean and unambiguous.

We have described the amplitude of the initial vector $y(0)$ but not its direction. For Fig. 4 and the other analogous plots of this paper, $y(0)$ is always determined by the following recipe:

$$
y(0)=C\left(y_{\max }+0.1 y_{\text {rand }}\right)
$$

Here, $y_{\max }$ denotes the unit vector that grows most rapidly at $t=0$, that is, with $\left\|y_{\max }\right\|=1$ and $(d / d t)\left\|e^{t A} y_{\max }\right\|$ at $t=0$ as large as possible (obtained via the eigenvalue decomposition of $\left.\left(A+A^{*}\right) / 2\right)$. The vector $y_{\text {rand }}$ is a unit vector whose entries are first taken as independent samples from the standard normal distribution, then rescaled by a constant so that $\left\|y_{\text {rand }}\right\|=1$. Thus $y(0)$ consists of a vector designed to excite great linear growth plus a noise vector of relative size $10 \%$ (by amplitude) or $1 \%$ (by energy). Such noise is necessary in some of our lowdimensional models - as in the Navier-Stokes equations themselves - to break symmetries associated with structures independent of the streamwise or spanwise coordinate. Its initial amplitude has little effect on the overall behavior.

Though we present only one figure for each model described in this paper, several runs have in fact been made in each case, with distinct vectors $y_{\text {rand }}$, to ensure that the behavior in the plot presented is typical.

In summary: for our first model under consideration, (TTRD), a bootstrapping phenomenon occurs and the threshold exponent is $\alpha=-3$.

\section{OTHER CORNELL MODELS}

Equation (TTRD) has been criticized on the grounds that although the norm $\|\cdot\|$ may be appealingly simple, the Navier-Stokes equations contain quadratic products of variables, not norms. It is easy to modify (TTRD) so that it will have this property. For example, we may consider

$$
\left(\begin{array}{l}
u \\
v
\end{array}\right)^{\prime}=\left(\begin{array}{cc}
-R^{-1} & 1 \\
-R^{-1}
\end{array}\right)\left(\begin{array}{l}
u \\
v
\end{array}\right)+\left(\begin{array}{c}
-u \\
u
\end{array}\right)\left(\begin{array}{l}
u \\
v
\end{array}\right)
$$

or

$$
\left(\begin{array}{l}
u \\
v
\end{array}\right)^{\prime}=\left(\begin{array}{rr}
-R^{-1} & 1 \\
-R^{-1}
\end{array}\right)\left(\begin{array}{l}
u \\
v
\end{array}\right)+\left(\begin{array}{c}
-v \\
v
\end{array}\right)\left(\begin{array}{l}
u \\
v
\end{array}\right) .
$$

Figs. 5a-b show the evolution of $\left(\mathrm{TTRD}^{\prime}\right)$ and $\left(\mathrm{TTRD}^{\prime \prime}\right)$ for various initial amplitudes. The first looks almost exactly the same as Fig. 4 for amplitudes $\|y\| \ll 1$; again 


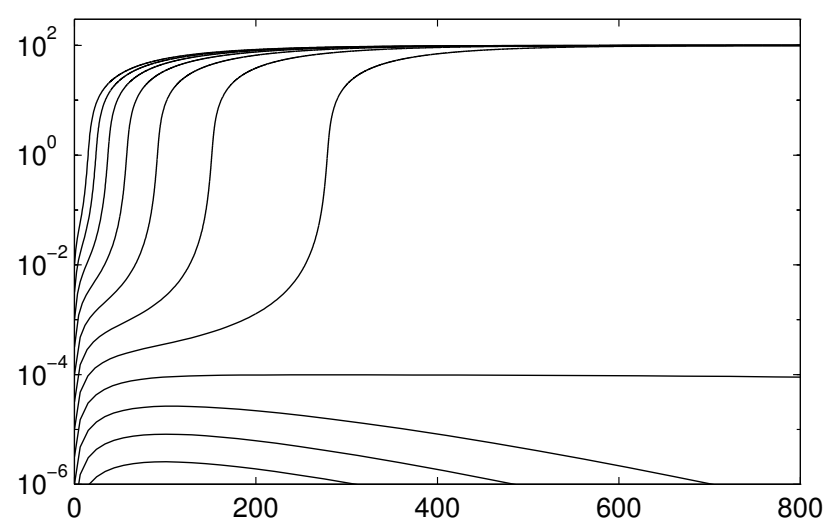

(a) Model (TTRD') (threshold exponent $\alpha=-3$ )

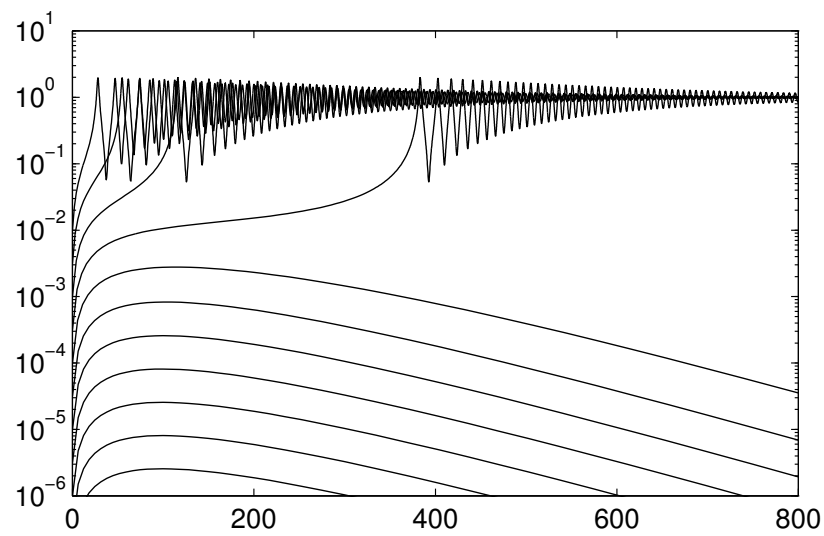

(b) Model (TTRD") (threshold exponent $\alpha=-2$ )

FIG. 5. Evolution curves for two other two-variable models with $R=100$.

the threshold exponent is $\alpha=-3$. The second looks qualitatively similar, but now the bootstrapping effect is evidently weaker, and in fact, we have $\alpha=-2$. In Sec. 9 we shall explain why these different exponents appear.

A second criticism of (TTRD) has been that if the matrix in the nonlinear term is chosen at random, though still skew-symmetric, then transition is observed with probability only 0.5 . The other half of the time, the nonlinear mixing rotates energy in the phase plane in the wrong direction, shutting off the loop of Fig. 3a. One might think that this shut-off effect calls into question the idea that arbitrary nonlinear mixing is likely to generate a phenomenon of subcritical transition. In fact, it is an artifact of the triviality of two-dimensional dynamics. To elucidate this point, a three-variable analogue of (TTRD) was proposed in [1]:

$$
\begin{aligned}
\left(\begin{array}{c}
u \\
v \\
w
\end{array}\right)^{\prime}= & \left(\begin{array}{ccc}
-2 R^{-1} & \beta(R) & \\
& -2 R^{-1} & \beta(R) \\
& -2 R^{-1}
\end{array}\right)\left(\begin{array}{c}
u \\
v \\
w
\end{array}\right) \\
& +\left\|\left(\begin{array}{c}
u \\
v \\
w
\end{array}\right)\right\|\left(\begin{array}{ccc}
a & a & b \\
-a & & c \\
-b & -c &
\end{array}\right)\left(\begin{array}{c}
u \\
v \\
w
\end{array}\right) .
\end{aligned}
$$

As in (TTRD), the linear term here introduces transient, non-modal amplification of amplitude $O(R)$ and

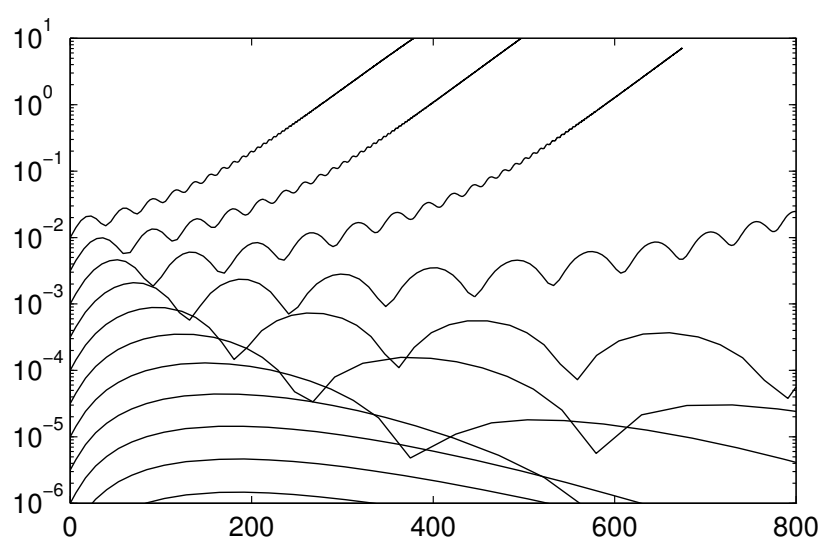

FIG. 6. Evolution curves for model (BDT) (threshold exponent $\alpha=-3$ ) with $R=100$.

time scale $O(R)$; the value $\beta(R)=3.86 \sqrt{(R+1) / R^{2}}$ was chosen in [1] so that the " $O$ " constants are approximately 1. The entries $a, b, c$ in the nonlinear term were chosen arbitrarily, but scaled so that the matrix has norm 1 .

As expected, (4) differs from (TTRD) in that arbitrary choices of nonlinear coefficients yield transition with probability close to 1 , not 0.5 , for sufficiently high $R$. Depending on the choices of $a, b$, and $c$, the asymptotic ("turbulent") state may be a finite fixed point, the point at infinity, a limit cycle, or chaos. The early stages of transition are independent of the final state, and the threshold exponent is again $\alpha=-3$. These matters are discussed in detail in [1].

As before, for the purposes of this paper it is convenient to discard most details and consider simply

$$
\begin{aligned}
& \left(\begin{array}{c}
u \\
v \\
w
\end{array}\right)^{\prime}=\left(\begin{array}{ccc}
-R^{-1} & R^{-1 / 2} & \\
& -R^{-1} & R^{-1 / 2} \\
& & -R^{-1}
\end{array}\right)\left(\begin{array}{c}
u \\
v \\
w
\end{array}\right) \\
& +\left\|\left(\begin{array}{l}
u \\
v \\
w
\end{array}\right)\right\|\left(\begin{array}{rrr} 
& 1 & 1 \\
-1 & & 1 \\
-1 & -1 &
\end{array}\right)\left(\begin{array}{l}
u \\
v \\
w
\end{array}\right) .
\end{aligned}
$$

This system captures the essence of (4), though of course, since the constants $a, b, c$ have been set to 1 , it exhibits only a single behavior as $t \rightarrow \infty$ for any fixed choice of initial data. Fig. 6 shows the evolution of (BDT) in the usual format. Again we have a bootstrapping phenomenon, and the threshold exponent is $\alpha=-3$.

A third criticism of (TTRD) and (BDT) has been that in the Navier-Stokes equations, "nonlinear mixing" must take place in a more indirect fashion than in these models. In plane Couette flow, for example, the strongest transient amplification is achieved by a vortex tilting mechanism acting on structures independent of the streamwise coordinate, in which a perturbation in the form of a streamwise roll advects low- and high-velocity fluid to relatively high- and low-velocity surroundings, respectively, where it shows up as a streamwise streak. If the initial perturbation is perfectly independent of the streamwise coordinate, with zero energy in Fourier components corresponding to streamwise variation, then this 
situation must persist for all time and turbulence cannot be achieved. Algebraically, we can see this by noting that the nonlinear interactions of the Navier-Stokes equations operate in triads, obeying "selection rules" of the form

$$
\left( \pm \alpha_{1}, \pm \beta_{1}\right)+\left( \pm \alpha_{2}, \pm \beta_{2}\right) \rightarrow\left( \pm \alpha_{1} \pm \alpha_{2}, \pm \beta_{1} \pm \beta_{2}\right)
$$

where $\alpha$ and $\beta$ denote Fourier parameters in the streamwise and spanwise directions, respectively. In particular, a single mode $(\alpha, \beta)$ does not affect itself nonlinearly. Because (TTRD) and (BDT) do not incorporate such selection rules, one might expect that the Navier-Stokes equations should exhibit a weaker bootstrapping effect than these models. This possibility was mentioned in [1] and [23], where it was suggested that $\alpha$ may not be as low as -3 for actual fluid flows.

Curiously, a roughly equivalent restatement of the above criticism is that the models (TTRD) and (BDT) possess an unphysical property: they undergo "transition" even when the initial vector is uncontaminated by noise. In these models, an initial vector $y(0)$ determined solely on the basis of linear algebra considerations is enough to excite transition. We confirm this by experiments (not shown) that reveal that if the noise component of (3) is removed, the curves of Figs. 4-6 change very little.

It is a straightforward matter to modify (TTRD) and (BDT) to incorporate selection rules. In unpublished work in early 1995, we replaced the single pair of variables of (TTRD) by three pairs of variables. The resulting six-variable model can be written

$$
y^{\prime}=A y+B(y) y
$$

with

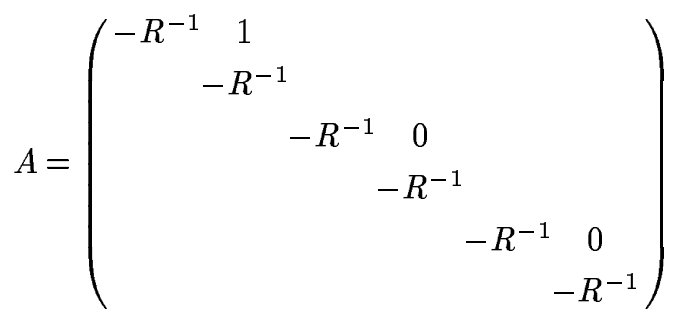

and

$$
B=\left(\begin{array}{ccc}
0 & y_{5} B_{5}+y_{6} B_{6} & y_{3} B_{3}+y_{4} B_{4} \\
-y_{5} B_{5}^{T}-y_{6} B_{6}^{T} & 0 & y_{1} B_{1}+y_{2} B_{2} \\
-y_{3} B_{3}^{T}-y_{4} B_{4}^{T} & -y_{1} B_{1}^{T}-y_{2} B_{2}^{T} & 0
\end{array}\right),
$$

where $B_{1}, \ldots, B_{6}$ are arbitrarily chosen $2 \times 2$ matrices with $\left\|B_{j}\right\|=1$. Fig. 7 shows evolution curves for (BT). As expected, the bootstrapping effect is weakened. However, it is still present: we have $\alpha=-2$, not $\alpha=-1$. This is readily explained; see Sec. 9. Again there is little qualitative dependence on the nonlinear coefficients: one gets transition with probability close to 1 . As expected, however, this is our first model for which transition fails to occur if the noise term of (3) is removed.

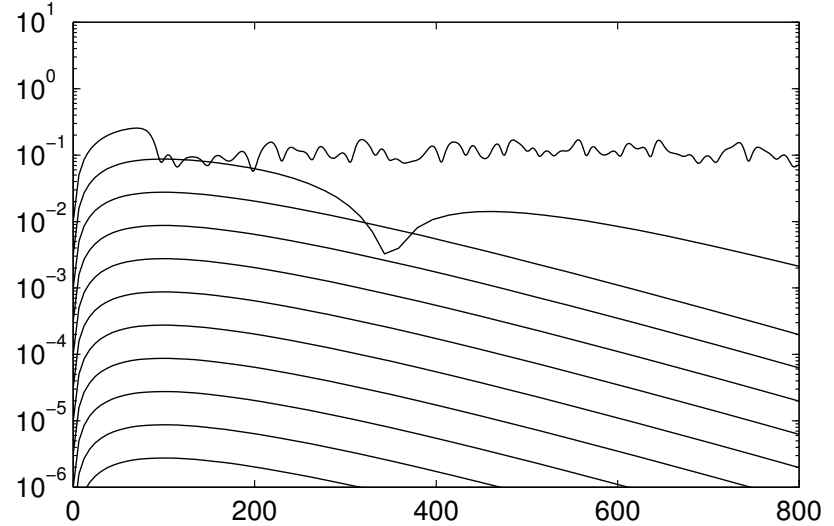

FIG. 7. Evolution curves for model (BT) (threshold exponent $\alpha=-2$ ) with $R=100$. These curves are based upon one particular (random) choice of the matrices $B_{j}$ in (7).

We have now presented plots of $\|y(t)\|$ vs. $t$ for five low-dimensional models. Do all the figures look more or less alike, at least for amplitudes $\|y\| \ll 1$ ? That, of course, is precisely the point. Although the various criticisms of (TTRD) that we have mentioned were all motivated by valid observations about fluid flows, none of them bear upon the qualitative behavior of these systems of equations for low disturbance amplitudes or the phenomenon of subcritical transition with $\alpha<-1$.

\section{MIT MODEL}

We now turn to a model that was conceived in an entirely different manner. This is the model proposed by Waleffe in [24] and [25]. It arose from studies of turbulence, not stability: specifically, from investigation of the generation and regeneration of streaks near boundaries in turbulent flows. In Waleffe's thinking, the essence of his model is distinctly nonlinear.

Waleffe's work in this area began around 1990 in collaboration with Kim and Hamilton at the Center for Turbulence Research. The questions that led to it were some of those that have been important in studies of turbulence, such as, what determines the spacing of streaks in turbulent boundary layers? As in the recent studies of hydrodynamic stability, it was recognized from the start that the mechanism of streak generation was linear [16]. But the emphasis in this work was on the search for a nonlinear mechanism that might produce a "selfsustaining process" whereby streaks could form, decay, and form again.

In [10] and [26], Waleffe and his colleagues proposed the self-sustaining process schematized in Fig. 3b. In the upper-left portion of the loop in that figure, streamwise vorticity generates streamwise streaks by linear advection. These streaks then break down according to an instability that can be viewed as linear and modal, essentially the Kelvin-Helmholtz instability. Finally, nonlinear interactions occur that recreate streamwise vorticity, and the process continues.

The cycle of Fig. 3b is not formulated as an ordinary 
differential equations model in [10] and [26]. In reaction to [23], however, Waleffe wrote the paper [24], in which he did formulate his views in this way. Upon publication of [1], he wrote a second paper [25] in which the model was generalized to include Reynolds number dependence. This model, containing four variables, takes the form

$$
\begin{aligned}
& \left(\begin{array}{c}
u \\
v \\
w
\end{array}\right)^{\prime}=\left(\begin{array}{ccc}
-\lambda R^{-1} & m & \\
& -\mu R^{-1} & \\
& & -\nu R^{-1}
\end{array}\right)\left(\begin{array}{l}
u \\
v \\
w
\end{array}\right) \\
& +\left(\begin{array}{rr}
-\gamma w \\
\delta w \\
\gamma w-\delta w &
\end{array}\right)\left(\begin{array}{c}
u \\
v \\
w
\end{array}\right), \\
& m^{\prime}=-\sigma R^{-1} m+\sigma R^{-1}-u v,
\end{aligned}
$$

where $\lambda, \mu, \nu, \sigma, \gamma, \delta$ are positive constants of order 1 . Unlike the variables in the models of the last two sections, the variables in (8) are given physical interpretations from the start. Roughly, they represent amplitudes of a streamwise streak $(u)$, a streamwise vortex $(v)$, a streamwise undulation of the streak that renders it unstable $(w)$, and the mean shear amplitude $(m)$. For a laminar flow, the values are $u=v=w=0$ and $m=1$.

As written above, Waleffe's equations do not exhibit a separation into linear and quadratic terms; the entry $m$ in the first matrix and the inhomogeneous term $R^{-1}$ in the scalar equation break the pattern. However, this is an artifact of a choice of variables in which the laminar flow corresponds to $m \neq 0$. To regularize the situation, let us define $n=m-1$. The equations become

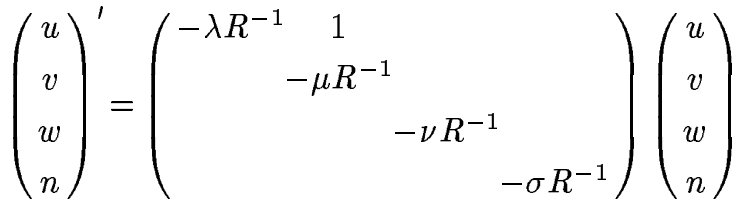

$$
\begin{aligned}
& +\left(\begin{array}{cccc}
n & -\gamma w & 0 \\
\gamma w & -\delta w & & \\
-v & & &
\end{array}\right)\left(\begin{array}{l}
u \\
v \\
w \\
n
\end{array}\right) .
\end{aligned}
$$

Waleffe does not discuss the threshold exponent exhibited by this model, although he suggests that models which give $\alpha<-1$ are likely to be nonphysical [25]. We have computed $\alpha$ for (W) and find that it is -2 . The evolution curves are shown in Fig. 8 for the arbitrary choice $\lambda=\mu=\nu=\sigma=\gamma=\delta=1$.

For the study of turbulence, since the perturbations of the laminar state involved are of order 1, an important feature of Waleffe's model is the energy balance reflected in the variable $n$. For the study of threshold exponents $\alpha<-1$ for transition, however, it is the behavior of low-amplitude perturbations that dominates. The energy balance is of little consequence so long as $\alpha<-1$, and $n$ can be replaced by zero. Since our concern in this

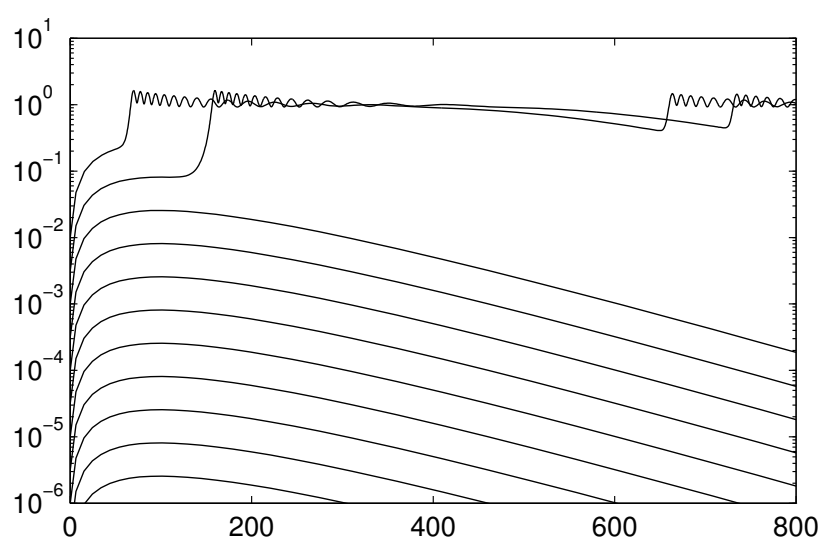

FIG. 8. Evolution curves for model (W) (threshold exponent $\alpha=-2$ ) with $R=100$.

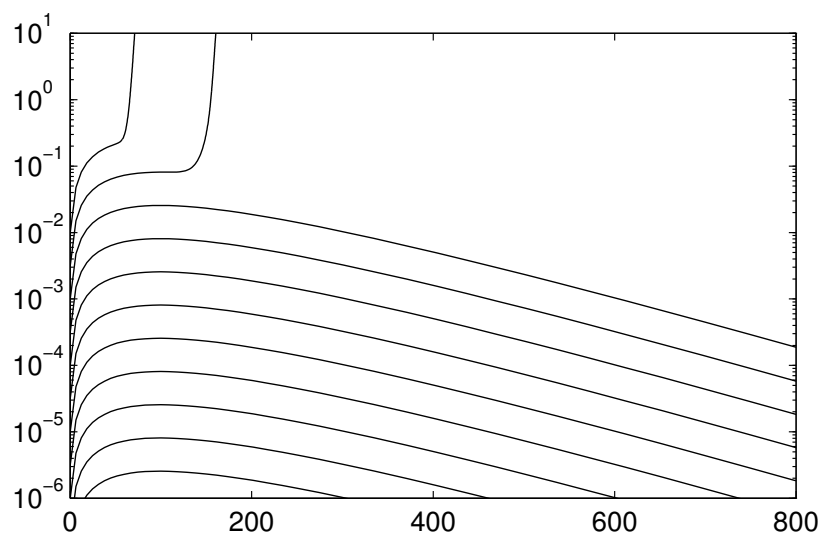

FIG. 9. Evolution curves for model ( $\left.\mathrm{W}^{\prime}\right)$ (threshold exponent $\alpha=-2$ ) with $R=100$.

paper is qualitative behavior rather than quantitative details, we may also replace the constants $\lambda, \mu, \nu, \sigma, \gamma, \delta$ by 1 . Now the equations reduce to a three-variable system:

$$
\begin{aligned}
\left(\begin{array}{c}
u \\
v \\
w
\end{array}\right)^{\prime}= & \left(\begin{array}{rrr}
-R^{-1} & 1 & \\
& -R^{-1} & \\
& & -R^{-1}
\end{array}\right)\left(\begin{array}{l}
u \\
v \\
w
\end{array}\right) \\
& +\left(\begin{array}{rr}
-w \\
w-w &
\end{array}\right)\left(\begin{array}{c}
u \\
v \\
w
\end{array}\right) .
\end{aligned}
$$

Fig. 9 shows the corresponding evolution curves. The global behavior is entirely different from that of (W), but the low-amplitude behavior is almost the same, and again the threshold exponent is $\alpha=-2$.

\section{MARBURG MODEL}

Now we cross the Atlantic to the fluid mechanics group of Siegfried Grossmann at the University of Marburg in Germany.

The papers $[4,9,19,23]$ cited above were a closely related series of works appearing in 1991-1993 on the subject of linear, non-normal effects in hydrodynamic stability. When these papers were written, their authors 
were unaware of a remarkable publication by Boberg and Brosa that had proposed many of the same ideas four years earlier [2]. In retrospect, it now appears that the schema of Fig. 3a was first described in the BobergBrosa paper. The Marburg group, like the Cornell group, have taken the view that more or less arbitrary nonlinear mixing will sufficient to induce transition in flows with non-normal linear amplification. Boberg and Brosa write bluntly, "The nonlinearity is a random mixer."

Though some low-dimensional models are discussed in [2], for example of dimensions 10 and 20, no single model is settled upon and no Reynolds number dependence is included. More recently, however, prompted by the two-dimensional model (TTRD), Gebhardt and Grossmann have published a paper in which they propose a model containing two complex variables [8]. This work is close to that of [1]; the two were independent and approximately simultaneous. To facilitate comparisons, we have we have rescaled Gebhardt and Grossmann's variables $t$ and $u$ (which we call $y$ ) by factors $R$ and $R^{-1}$, respectively, and then replaced $R$ by $R / 20$. Their equations now take the form

$$
y^{\prime}=A y+B(y, y)+C(\|y\|) y
$$

where

$$
\begin{gathered}
A=\left(\begin{array}{cc}
-.9 R^{-1}+.8 i \gamma & .7 \\
0 & -1.9 R^{-1}+1.1 i \gamma
\end{array}\right), \\
B_{1}(y, y)=.2 y_{1} y_{2}+.4 i y_{1} y_{2}^{*}+.6 y_{1}^{*} y_{2}+.8 i y_{1}^{*} y_{2}^{*} \\
-1.4 y_{2}^{2}-(1+1.2 i) y_{2} y_{2}^{*}-1.6 i y_{2}^{* 2}, \\
B_{2}(y, y)=y_{1} y_{2}+1.2 i y_{1}^{*} y_{2}+1.4 y_{1} y_{2}^{*}+1.6 i y_{1}^{*} y_{2}^{*} \\
-.6 y_{1}^{2}-(.2+.4 i) y_{1} y_{1}^{*}-.8 i y_{1}^{* 2},
\end{gathered}
$$

and

$$
C(\|y\|)=\frac{-\|y\|}{0.1+\|y\|}\left(\begin{array}{ll}
0 & .7 \\
0 & 0
\end{array}\right) .
$$

In these equations, the $A$ term represents linear nonmodal amplification, the $B$ term is quadratic and energyconserving (though this is not obvious as written), the $C$ term is a mean flow adjustment analogous to Waleffe's fourth equation in (8), and $\gamma$ is a zero or nonzero parameter corresponding to background convection.

These equations look quite different from those we have considered so far. However, let us rewrite them in terms of real variables. Setting $y=(u, v, w, x)=\left(\operatorname{Re} y_{1}\right.$, $\left.\operatorname{Im} y_{1}, \operatorname{Re} y_{2}, \operatorname{Im} y_{2}\right)$ and taking $\gamma=0$, we are led to the equation

$$
y^{\prime}=A y+B(y) y+C(\|y\|) y,
$$

with

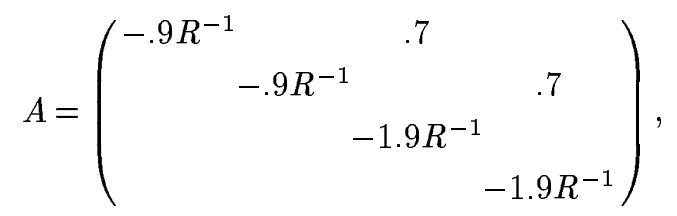

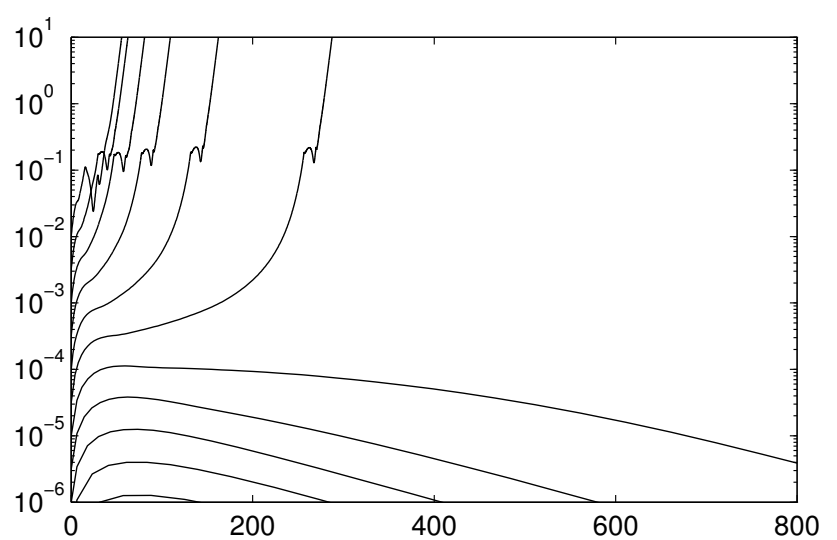

FIG. 10. Evolution curves for model (GG) (threshold exponent $\alpha=-3$ ) with $R=100$.

$$
\begin{aligned}
& B(y)=\frac{1}{5}\left(\begin{array}{cc}
0 & -x-2 w \\
x+2 w & 0 \\
-4 u+12 w-4 v+8 x & 2 v+7 x-4 u+14 w \\
-3 v-2 x-6 u+8 w & -3 u+7 w+2 v-2 x
\end{array}\right. \\
& \left.\ldots \begin{array}{cc}
4 u-12 w+4 v-8 x & 3 v+2 x+6 u-8 w \\
-2 v-7 x+4 u-14 w & 3 u-7 w-2 v+2 x \\
0 & -5 v-6 u \\
5 v+6 u & 0
\end{array}\right),
\end{aligned}
$$

and

$$
C(\|y\|)=\frac{-\|y\|}{0.1+\|y\|}\left(\begin{array}{lll} 
& .7 & \\
& & .7 \\
& &
\end{array}\right) .
$$

Note that $B(y)$ is skew-symmetric, explaining why this term is energy-conserving.

The complicated entries of $B(y)$ in this model can be viewed as essentially random; these entries were selected arbitrarily by Gebhardt and Grossmann. In [8] there is no discussion of the exponent $\alpha$, though there is some consideration of threshold amplitudes near Figs. 6 and 8. For a model of this kind with no selection rules, one would expect the exponent to be $\alpha=-3$. This expectation is confirmed by experiments (Fig. 10).

\section{STOCKHOLM MODEL}

Numerous contributions have been made to questions of transition over the years by a group of researchers whom we may loosely associate with the Royal Institute of Technology in Stockholm. Among the central figures in the recent work are Dan Henningson, who was also at MIT for a time, and Peter Schmid, his student at MIT.

In 1992, Henningson and Schmid proposed a threevariable model of certain features of transition to turbulence, though without Reynolds number dependence [11]. This model involved three eigenmodes, and the linear term was accordingly diagonal; this was before this group became fully involved in the non-normal developments of $[2,4,9,19,23]$. Rather than giving further de- 


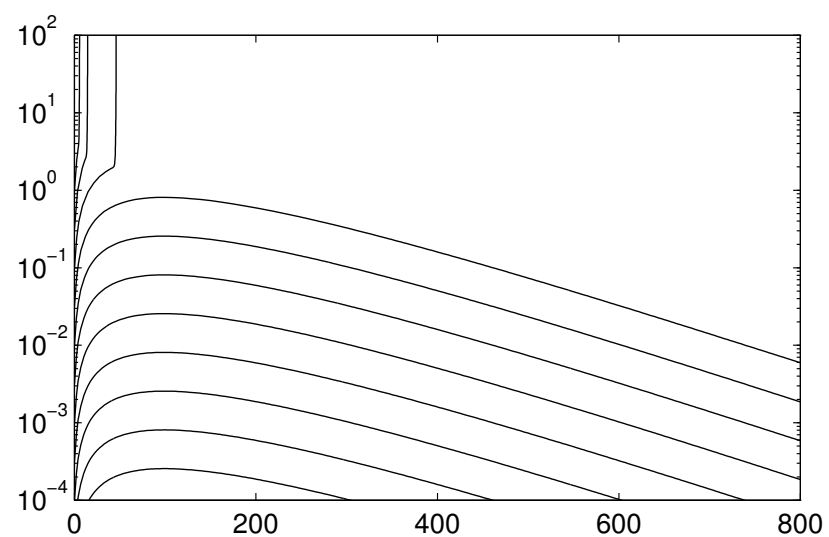

FIG. 11. Evolution curves for model (KLH) (threshold exponent $\alpha=-1$ ) with $R=100$. This is the only figure in this in this paper in which no bootstrapping appears. Note that the vertical scale is shifted by two orders of magnitude; the initial amplitudes have been correspondingly shifted to $\|y(0)\|=10^{-5}, \ldots, 10^{0}$.

tails, let us turn to a later model proposed by G. Kreiss, Lundbladh, and Henningson. This model appears in a paper explicitly devoted to the question of threshold exponents [15]. Its origin is rather different from the other models we have discussed, however, for besides being motivated by the Navier-Stokes equations themselves, it is also designed to illustrate certain points regarding the mathematical techniques used in that paper.

The KLH model is as follows:

$$
\left(\begin{array}{c}
u \\
v \\
w
\end{array}\right)^{\prime}=\left(\begin{array}{rrr}
-R^{-1} & 1 & \\
-R^{-1} & \\
& & -1
\end{array}\right)\left(\begin{array}{l}
u \\
v \\
w
\end{array}\right)+\left(\begin{array}{c}
w^{2} \\
u w
\end{array}\right)
$$

Here $u$ represents a streamwise streak, $v$ a streamwise vortex, and $w$ a non-streamwise mode of some kind. The nonlinear term is intended to be suggestive of certain processes without capturing them accurately, and energy conservation is intentionally not included in the model.

Like the other models we have considered, (KLH) contains a non-normal matrix as its linear term. The threshold exponent for transition turns out to be $\alpha=-1$, the first time we have encountered a value that is not $<-1$. See Fig. 11 .

The reason for the exponent $\alpha=-1$ is easily spotted. The $(3,3)$ entry of the linear term of $(\mathrm{KLH})$ is -1 , not $-R^{-1}$. This term represents convective decay, and the entry -1 corresponds to a decay rate independent of $R$. If one modifies this part of the model to allow a diminishing decay rate as $R \rightarrow \infty$, one obtains a model such as

$$
\left(\begin{array}{l}
u \\
v \\
w
\end{array}\right)^{\prime}=\left(\begin{array}{ccc}
-R^{-1} & 1 & \\
& -R^{-1} & \\
& & -R^{-\sigma}
\end{array}\right)\left(\begin{array}{l}
u \\
v \\
w
\end{array}\right)+\left(\begin{array}{c}
w^{2} \\
u w
\end{array}\right)
$$

for some $\sigma$ presumably in the range $-1 \leq \sigma<0$. As we shall explain in Sec. 9, the threshold exponent becomes $\alpha=-1-\sigma$. The corresponding energy history is plotted in Fig. 12 for the case $\sigma=1$. Note the strong resemblance to Fig. 9 ; eqs. $\left(\mathrm{W}^{\prime}\right)$ and $\left(\mathrm{KLH}^{\prime}\right)$ are almost the same.

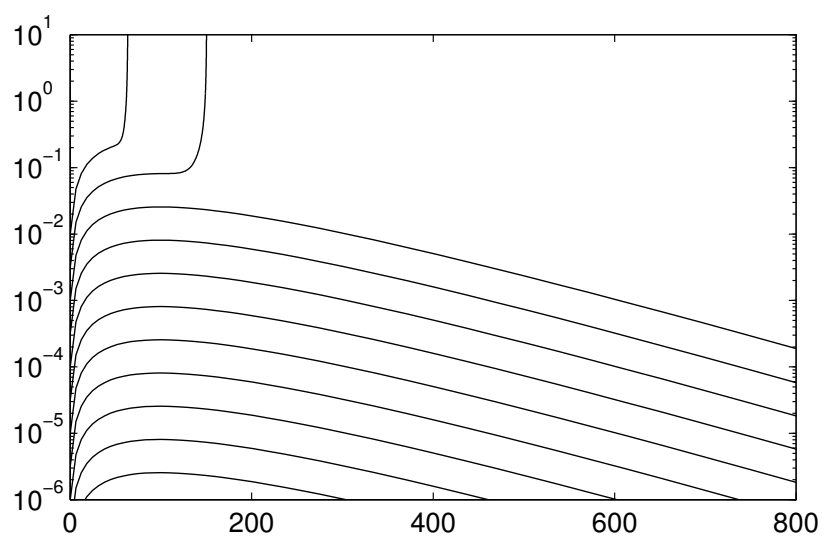

FIG. 12. Evolution curves for model $\left(\mathrm{KLH}^{\prime}\right)$ (threshold exponent $\alpha=-2$ ) with $\sigma=1, R=100$.

\section{GÖTEBORG MODEL}

Not far away in Sweden, another group has been active at the Chalmers Institute of Technology in Göteborg. The manuscript [13], by Claes Johnson and Mats Boman of Chalmers together with Rolf Rannacher of the University of Heidelberg, proposes a three-variable ODE model. The motivation for this model is tied to computational fluid mechanics: these researchers are concerned with error control in numerical simulations, a challenging problem since small perturbations may have large and long-lasting effects.

Like Waleffe's equations (8), the equations of [13] are not written in our standard linear-plus-quadratic form. However, by replacing their variable $u_{1}-1$ by $u$, we can remedy this. To facilitate comparisons we also rename other variables of [13] as follows: $R=\nu^{-1}, v=u_{2}, w=u_{3}$, $a=\gamma_{12}, b=\gamma_{13}, c=\gamma_{31}$. The equations become

$$
\left(\begin{array}{c}
u \\
v \\
w
\end{array}\right)^{\prime}=\left(\begin{array}{ccc}
-R^{-1} & a & b \\
-R^{-1} & \\
& & -R^{-1}
\end{array}\right)\left(\begin{array}{l}
u \\
v \\
w
\end{array}\right)+\left(\begin{array}{c}
a u v+b u w \\
0 \\
c R^{-1} u w
\end{array}\right)
$$

where $a, b, c$ are nonnegative constants. Note that the variable $v$ in this system evolves linearly, unaffected by the other variables. For $t \rightarrow \infty,(\mathrm{JRB})$ is in fact close to the two-variable model $\left(\mathrm{TTRD}^{\prime \prime}\right)$, except that the quadratic coupling term $c R^{-1} u w$ has coefficient $O\left(R^{-1}\right)$ instead of $O(1)$. For this reason, the threshold exponent turns out to be $\alpha=-1$ (figure not shown).

\section{WHY THESE FIGURES ALL LOOK ALIKE: TWO VIEWS OF STREAK INSTABILITY}

We come now to the mathematical heart of this paper. Why do all the curves we have presented look so similar, at least as long as the amplitudes remain 
TTRD: $(\alpha=-3)$

$$
\left(\begin{array}{l}
u \\
v
\end{array}\right)^{\prime}=\left(\begin{array}{cc}
-R^{-1} & 1 \\
-R^{-1}
\end{array}\right)\left(\begin{array}{l}
u \\
v
\end{array}\right)+\left\|\left(\begin{array}{l}
u \\
v
\end{array}\right)\right\|\left(\begin{array}{l} 
\\
u
\end{array}\right)
$$

$\mathrm{TTRD}^{\prime}:(\alpha=-3)$

$$
\left(\begin{array}{l}
u \\
v
\end{array}\right)^{\prime}=\left(\begin{array}{cc}
-R^{-1} & 1 \\
-R^{-1}
\end{array}\right)\left(\begin{array}{l}
u \\
v
\end{array}\right)+\left(\begin{array}{c} 
\\
u^{2}
\end{array}\right)
$$

TTRD $^{\prime \prime}:(\alpha=-2)$

$$
\left(\begin{array}{l}
u \\
v
\end{array}\right)^{\prime}=\left(\begin{array}{cc}
-R^{-1} & 1 \\
-R^{-1}
\end{array}\right)\left(\begin{array}{l}
u \\
v
\end{array}\right)+\left(\begin{array}{c}
u \\
u v
\end{array}\right)
$$

BDT: $(\alpha=-3)$

$\left(\begin{array}{c}u \\ v \\ w\end{array}\right)^{\prime}=\left(\begin{array}{rrr}-R^{-1} & R^{-1 / 2} & \\ & -R^{-1} & R^{-1 / 2} \\ & & -R^{-1}\end{array}\right)\left(\begin{array}{c}u \\ v \\ w\end{array}\right)+\left\|\left(\begin{array}{c}u \\ v \\ w\end{array}\right)\right\|\left(\begin{array}{l} \\ u\end{array}\right)$

$\mathrm{W}^{\prime}:(\alpha=-2)$

$\left(\begin{array}{c}u \\ v \\ w\end{array}\right)^{\prime}=\left(\begin{array}{ccc}-R^{-1} & 1 & \\ -R^{-1} & \\ & & -R^{-1}\end{array}\right)\left(\begin{array}{c}u \\ v \\ w\end{array}\right)+\left(\begin{array}{c}w^{2} \\ u w\end{array}\right)$.

$\mathrm{KLH}^{\prime}:(\alpha=-1-\sigma)$

$$
\left(\begin{array}{c}
u \\
v \\
w
\end{array}\right)^{\prime}=\left(\begin{array}{ccc}
-R^{-1} & 1 & \\
& -R^{-1} & \\
& & -R^{-\sigma}
\end{array}\right)\left(\begin{array}{c}
u \\
v \\
w
\end{array}\right)+\left(\begin{array}{c}
w^{2} \\
u w
\end{array}\right)
$$

Table 1. Stripped-down low-dimensional models. The models have been simplified by elimination of all terms that do not affect the threshold exponent $\alpha$.

$\ll 1$ ? The answer was summarized in the caption of Fig. 3. The Cornell and Marburg groups speak of "nonlinear mixing," and the MIT and Swedish groups speak of "streak instabilities" and other physical notions, but these are different ways of looking at the same phenomena. In a system exhibiting linear, non-modal transient growth, more or less arbitrary nonlinearities may produce a "streak instability;" one does not have to put it in the model specifically.

To explain this statement, let us first of all take the final step in removing extraneous details from some of our models. Most of the differences in nonlinear terms between the various equations we have presented result from different notions of how to handle energy conservation, and relatedly, modification of the mean flow by finite-amplitude perturbations. However, these distinctions are significant only for amplitudes $O(1)$; they have no effect on the threshold exponent $\alpha$ if $\alpha<-1$. Thus: let us remove them. Table 1 summarizes some of the models we have discussed, but with nonlinear terms removed that do not affect $\alpha$.

We now present heuristic arguments that explain the threshold exponents we have observed. Each of these models contains a linear, non-modal amplifier of gain $O(R)$ and time scale $O(R)$. Consider the following caricature. The output of the amplifier (the relatively highamplitude quantity in the early stages of a process of transition) is a variable $\varphi(t)$. All other quantities of interest, including the input to the amplifier, are represented by another variable $\psi(t)$ (potentially of much lower amplitude in the early stages of transition). Both $\varphi$ and $\psi$ start with amplitude $\epsilon$. The variable $\varphi(t)$ grows to size $\epsilon R$ by linear, non-modal effects, and lingers at that level for a time $O(R)$. The variable $\psi$ grows by quadratic interactions,

$$
\psi^{\prime} \approx \varphi \psi, \quad \psi(0) \approx \epsilon
$$

hence for a period of duration $O(R)$,

$$
\psi^{\prime} \approx \epsilon R \psi, \quad \psi(0) \approx \epsilon
$$

with solution

$$
\psi(t) \approx \epsilon e^{\epsilon R t}, \quad 0 \leq t \leq O(R)
$$

All together, we predict exponential growth at rate $\epsilon R$ up to an amplitude of order $\epsilon e^{\epsilon R^{2}}$. Unless $\epsilon R^{2}$ is bounded, this corresponds to growth by an arbitrary factor, so that $\psi$ may achieve amplitude $O(1)$, after which anything may happen. In other words, the threshold exponent for "transition" in (9)-(11) is $\alpha=-2$.

Models (TTRD $\left.{ }^{\prime \prime}\right)$, (BT), (W), and ( $\left.\mathrm{W}^{\prime}\right)$ fit the pattern just described. For (TTRD ${ }^{\prime \prime}$ ), this is obvious; the variables $u$ and $v$ play the roles of $\varphi$ and $\psi$, respectively. For the others, the variable $u$ (or $y_{1}$ in the case of (BT)) plays the role of $\varphi$, and all the other variables collectively correspond to $\psi$. In the case of $\left(\mathrm{W}^{\prime}\right)$, for example, the term $u w$ represents the quadratic interaction $\varphi \psi$ of (9), feeding energy back from the output of the linear amplifier $(u)$ into the rest of the system ( $v$ and $w$ ).

In (9), there is no quadratic term $\varphi^{2}$. The absence of this term is the representation within (9)-(11) of the crucial "selection rule" of the kind discussed earlier. Physically, we may think of the fact that a purely streamwise streak, no matter how large in amplitude, cannot by itself feed energy into modes that are not independent of the streamwise coordinate. By contrast, if we have a system with no such selection rule, we can caricature it by changing $\varphi \psi$ to $\varphi^{2}$ in (9). Equations (9)-(11) become

$$
\begin{gathered}
\psi^{\prime} \approx \varphi^{2}, \quad \psi(0) \approx \epsilon, \\
\psi^{\prime} \approx(\epsilon R)^{2}, \quad \psi(0) \approx \epsilon, \\
\psi(t) \approx(\epsilon R)^{2} t, \quad 0 \leq t \leq O(R),
\end{gathered}
$$

leading to $\psi(t)=O\left(\epsilon^{2} R^{3}\right)$ at $t=O(R)$. Now, if $\epsilon^{2} R^{3}$ is of order $\epsilon$ or greater, then $\psi$ may be larger at $t=O(R)$ than it was at $t=0$. Another round of amplification at a higher level may begin, leading to self-sustaining growth up to amplitude $O(1)$. In other words, the threshold exponent for "transition" in (12)-(14) is determined by the 
condition $\epsilon=\epsilon^{2} R^{3}$, giving $\alpha=-3$ [23]. Models (TTRD ${ }^{\prime}$ ) and (GG) fit this pattern. So do models (TTRD) and (BDT), though the quadratic nature of the nonlinearity is obscured by the use of the norm $\|y\|$.

We remark that the question of whether or not a model incorporates selection rules is not as black-andwhite as the above discussion may suggest. For example, we mentioned that $\left(\mathrm{TTRD}^{\prime \prime}\right),(\mathrm{BT}),(\mathrm{W})$, and $\left(\mathrm{W}^{\prime}\right)$ all contain the crucial selection rule that no $\varphi^{2}$ term is present. However, the last three of these carry the idea further than the first by incorporating an additional selection rule within the group of variables corresponding to $\psi$. Specificially (in the notation of $(\mathrm{W})$ and $\left(\mathrm{W}^{\prime}\right)$ ), $u$ does not affect $v$ directly, but only indirectly through $w$. As a result, though the final threshold exponent $\alpha$ is not affected, (BT) and (W) and $\left(\mathrm{W}^{\prime}\right)$ require "noise" in the form of an initial value $w \neq 0$ to excite transition, whereas (TTRD") has no such requirement.

Easy modifications of these arguments explain why our remaining three models, $(\mathrm{KLH})$ and $\left(\mathrm{KLH}^{\prime}\right)$ and (JRB), have threshold exponents $\alpha=-1,-1-\sigma$, and -1 , respectively.

The two-variable caricature (9)-(11) has more physics in it than one might expect. In this pair of equations, the presence of an approximately steady high-amplitude signal $\varphi(t)$ makes it possible for a second signal $\psi(t)$ to grow exponentially over many orders of magnitude. This is nothing more than an abstract description of a linear secondary instability. In the case of the breakdown of a streamwise streak, $\varphi$ corresponds to the amplitude of the streak and $\psi$ to, among other quantities, the amplitude of a deviation from perfect streamwise independence. The exponential growth of such deviations, just as in (11), appears strikingly in certain Navier-Stokes simulations of transition to turbulence from initial perturbations consisting of streamwise vortices plus noise. Note that (10)-(11) predict that the growth rate of the instability should be proportional to the amplitude of the streak. Under certain circumstances, though not all, exactly this behavior is observed in Navier-Stokes simulations [20].

\section{DISCUSSION}

The compressed discussions of this paper cannot begin to do justice to the wide range of physical ideas put forward by the more than a dozen authors whose works we have compared. Mathematically, however, the low-dimensional models proposed by these authors have much in common. All are small systems of ordinary differential equations combining a non-normal linear term with a quadratic nonlinearity. In each case the zero solution is mathematically stable, but small perturbations can be amplified by factors $O(R)$ on a time scale $O(R)$. In most cases the nonlinearities are such that a "bootstrapping" phenomenon may occur, causing the threshold exponent for "transition" to be strictly less than -1 .

For actual fluid flows, the evidence concerning thresh- old exponents is sparse. What does seem clear, based on Navier-Stokes simulations, is that $\alpha$ is strictly less than -1 for for plane Couette flow $(\alpha \leq-5 / 4$ ?) and plane Poiseuille flow $(\alpha \leq-7 / 4$ ?) [12,17]. (For pipe Poiseuille flow, there are as yet no computations concerning threshold exponents.) In principle the actual exponents might be much less than these estimates, but numerical experiments by Baggett, Henningson, Lundbladh, Reddy, and Schmid have revealed no evidence of this so far. In view of this experimental situation and the fact that the Navier-Stokes equations contain selection rules of the kind we have discussed, a reasonable guess may be that for actual flows in pipes and channels, the true values of $\alpha$ lie in the range $-2 \leq \alpha<-1$.

\section{ACKNOWLEDGMENTS}

This work was supported by NSF Grant DMS-9500975CS and DOE Grant DE-FG02-94ER25199.

\section{REFERENCES}

[1] J. S. Baggett, T. A. Driscoll and L. N. Trefethen, "A mostly linear model of transition to turbulence," Phys. Fluids A 7, 833 (1995).

[2] L. Boberg and U. Brosa, "Onset of turbulence in a pipe," Z. Naturforschung 43a, 697 (1988).

[3] U. Brosa, "Turbulence without strange attractor," J. Stat. Phys. 55, 1303 (1989).

[4] K. M. Butler and B. F. Farrell, "Three-dimensional optimal perturbations in viscous shear flow," Phys. Fluids A 4, 1637 (1992).

[5] J. P. Crutchfield and K. Kaneko, "Are attractors relevant to turbulence?," Phys. Rev. Lett. 60, 2715 (1988).

[6] O. Dauchot and F. Daviaud, "Finite amplitude perturbation and spots growth mechanism in plane Couette flow," Phys. Fluids 7, 335 (1995).

[7] P. G. Drazin and W. H. Reid, Hydrodynamic Stability, Cambridge U. Press, Cambridge, 1981.

[8] T. Gebhardt and S. Grossmann, "Chaos transition despite linear stability," Phys. Rev. E 50, 3705 (1994).

[9] L. H. Gustavsson, "Energy growth of three-dimensional disturbances in plane Poiseuille flow," J. Fluid Mech. 224, 241 (1991).

[10] J. M. Hamilton, J. Kim, and F. Waleffe, "Regeneration mechanisms of near-wall turbulence structures," J. Fluid Mech. 287, 317 (1995).

[11] D. S. Henningson and P. J. Schmid, "Vector eigenfunction expansions for plane channel flows," Stud. Appl. Math. 87, 15 (1992).

[12] D. S. Henningson, "Bypass transition and linear growth mechanisms," in R. Benzi, ed., Advances in Turbulence V, Kluwer, 1995. 
[13] C. Johnson, R. Rannacher, and M. Boman, "On transition to turbulence and error control in CFD," preprint 1994:26, Dept. of Math., Chalmers Inst. of Tech., 1994.

[14] Lord Kelvin (W. Thomson), "Stability of fluid motion-rectilinear motion of viscous fluid between two parallel plates," Phil. Mag. 24, 188 (1887).

[15] G. Kreiss, A. Lundbladh, and D. S. Henningson, "Bounds for threshold amplitudes in subcritical shear flows," J. Fluid Mech. 270, 175 (1994).

[16] M. J. Lee, J. Kim, and P. Moin, "Structure of turbulence at high shear rate," J. Fluid Mech. 216, 561 (1990).

[17] A. Lundbladh, D. S. Henningson, and S. C. Reddy, "Threshold amplitudes for transition in channel flows," in Transition, Turbulence and Combustion, v. I, Kluwer, Dordrecht, 1994.

[18] A. Lundbladh and A. V. Johansson, "Direct simulation of turbulent spots in plane Couette flow," J. Fluid Mech. 229, 499 (1991).

[19] S. C. Reddy and D. S. Henningson, "Energy growth in viscous channel flows," J. Fluid Mech. 252, 209 (1993).

[20] S. C. Reddy, P. J. Schmid, and J. S. Baggett, "On the stability of streamwise streaks and transition to turbulence in channel flows," in preparation.

[21] P. J. Schmid and D. S. Henningson, "A new mechanism for rapid transition involving a pair of oblique waves," Phys. Fluids A 4, 1986 (1992).

[22] N. Tillmark and P. H. Alfredsson, "Experiments on transition in plane Couette flow," J. Fluid Mech. 235, 89 (1992).

[23] L. N. Trefethen, A. E. Trefethen, S. C. Reddy, and T. A. Driscoll, "Hydrodynamic stability without eigenvalues," Science 261, 578 (1993).

[24] F. Waleffe, "Hydrodynamic stability and turbulence: beyond transients to a self-sustaining process," Studies in Appl. Math. 95, 319 (1995).

[25] F. Waleffe, "Transition in shear flows. Non-linear normality versus non-normal linearity," Phys. Fluids 7, 3060 (1995).

[26] F. Waleffe, J. Kim, and J. Hamilton, "On the origin of streaks in turbulent shear flows," Turbulent Shear Flows 8, F. Durst et al., eds., Springer-Verlag, 1993, pp. 37-49. 